УДК 517.9

\title{
2D Thermocapillary Motion of Three Fluids in a Flat Channel
}

\author{
Victor K. Andreev* \\ Elena N. Cheremnykh ${ }^{\dagger}$ \\ Institute of computational modelling SB RAS \\ Akademgorodok, 50/44, Krasnoyarsk, 660036 \\ Institute of Mathematics and Computer Science \\ Siberian Federal University \\ Svobodny, 79, Krasnoyarsk, 660041
}

Russia

Received 17.05.2016, received in revised form 12.08.2016, accepted 15.09.2016

Two-dimensional creeping motion of three immiscible, incompressible viscous fluids in a flat channel bounded by fixed solid walls, on which the temperature distribution is known, is investigated. The motion is induced only by the thermalcapillary forces beginning from the state of rest. Unsteady motion is described by finite analytic formulas obtained by Laplace transform in images. The evolution of the velocity fields to the stationary regime for specific liquids is obtained by the numerical inversion of Laplace transformation.

Keywords: thermocapillarity, interface, mathematical modeling, numerical experiments.

DOI: 10.17516/1997-1397-2016-9-4-404-415.

It is well known that in a non-uniformly heated liquid a motion can arise. In some applications of liquid flows, a joint motion of two or more fluids with surfaces takes place. If the liquids are not soluble in each other, they form a more or less visual interfaces. The petroleum-water system is a typical example of this situation. At the present time modelling of multiphase flows taking into account different physical and chemical factors is needed for designing of cooling systems and power plants, in biomedicine, for studying the growth of crystals and films, in aerospace industry [1-4].

Nowadays, there are exact solutions of the Marangoni convection [5-7]. One of the first solutions was obtained in Napolitano [8]. This is the Poiseuille stationary flow of two immiscible liquids in an inclined channel. As a rule, all such flows were considered steady and unidirectional. The stability of such flows was investigated in $[9,10]$. As for non-stationary thermocapillary flows, studying of them began recently $[11,12]$.

Thermocapillary convection problem for two incompressible liquids separated by a closed interface in a container was investigated in [13]. Local (in time) unique solvability of the problem was obtained in Holder classes of functions. The problem of thermalcapillary $3 \mathrm{D}$ motion of a drop was studied in [14]. Moreover, its unique solvability in Holder spaces with a power-like weight at infinity was established. Velocity vector field decreases at infinity in the same way as the initial data and mass forces, the temperature diverges to the constant which is the limit of the initial temperature at infinity.

\footnotetext{
*andr@icm.krasu.ru

†elena_cher@icm.krasn.ru

(c) Siberian Federal University. All rights reserved
} 
The present work is devoted to studying of solutions of a conjugate boundary value problem arising as a result of linearization of the Navier-Stokes system asupplemented with temperature equation. The description of the $2 \mathrm{D}$ creeping joint motion of three viscous heat conducting fluids in flat layers is also provided here. The motion arises due to thermocapillary forces imposed along two interfaces, after which the unsteady Marangoni convection begins. Such kind of convection can dominate in flows under microgravity conditions or in motions of thin liquid films.

\section{Statement of problem}

The two-dimensional motion of three viscous incompressible heat conducting fluids in the absence of mass forces is described by the system

$$
\begin{gathered}
u_{t}+u u_{x}+v u_{y}+\frac{1}{\rho} p_{x}=\nu\left(u_{x x}+u_{y y}\right) \\
v_{t}+u v_{x}+v v_{y}+\frac{1}{\rho} p_{y}=\nu\left(v_{x x}+v_{y y}\right) \\
u_{x}+v_{y}=0 \\
\Theta_{t}+u \Theta_{x}+v \Theta_{y}=\chi\left(\Theta_{x x}+\Theta_{y y}\right)
\end{gathered}
$$

Here $u, v$ are the components of the velocity vector, $p$ is the pressure, $\Theta$ is the temperature, $\rho$ is the density, $\nu$ is the kinematic viscosity, $\chi$ is the thermal diffusivity. The values of $\rho, \nu, \chi$ are represented by constants.

We find the exact solution in the form

$$
u(x, y, t), v(y, t), p(x, y, t), \Theta(x, y, t)
$$

In such case, the first three equations of system (1) lead to the relations

$$
\begin{gathered}
u=w(y, t) x+u_{1}(y, t), \quad w+v_{y}=0, \\
w_{t}+v w_{y}+w^{2}=f(t)+\nu w_{y y}, \\
\frac{1}{\rho} p=d(y, t)-\frac{f(t)}{2} x^{2}, \\
d_{y}=\nu v_{y y}-v_{t}-v v_{y}, \quad u_{1 t}+v u_{1 y}+u_{1} w=0
\end{gathered}
$$

with an arbitrary function $f(t)$.

With respect to the temperature field we assume that it has the form

$$
\Theta=a(y, t) x^{2}+a_{1}(y, t) x+b(y, t) .
$$

It will be seen, Eq. (3) has a good agreement with the conditions at the interfaces.

The stationary solution of the Navier-Stokes equations in the form (2) for $g=0$ for pure viscous fluid was found for the first time by Hiemenz [15]. It describes the liquid inpingement from infinity on the plane $y=0$ under the no slip condition on it. In the paper Brady and Acrivos [16], this solution for the flow between two plates or for the flow in a cylindrical tube (axisymmetric analogue of solution (2)) was applied.

It is known that the temperature dependence of the surface tension coefficient is the one of the most important factors leading to the dynamic variety of the interfacial surface. In the 
papers Bobkov and Gupalo [17], Gupalo and Ryazantsev [18] the stationary solutions in form (2), (3) was found at $a(y, t) \equiv 0, b=$ const for a flat layer with a free boundary $y=l=$ const and a solid wall $y=0$. The non-uniqueness of solution depending on the physical parameters of the problem was revealed. A similar problem in the case of half space was investigated in Gupalo et al. [19].

Further, we assume that $u_{1}(y, t) \equiv 0, a_{1}(y, t) \equiv 0$. The latter means that the temperature field has an extremum at the point $x=0$, more precisely, at $a(y, t)<0$ it has a maximum and at $a(y, t)>0$ it has a minimum. Let us apply the solution in the form (2), (3) to describe the joint flow of three immiscible liquids in flat layer $0<y<l_{3}$ and take into account that the walls $y=0, y=l_{3}$ are solid (see Fig. 1). By introducing the index $j=1,2,3$ fixing the fluid and using

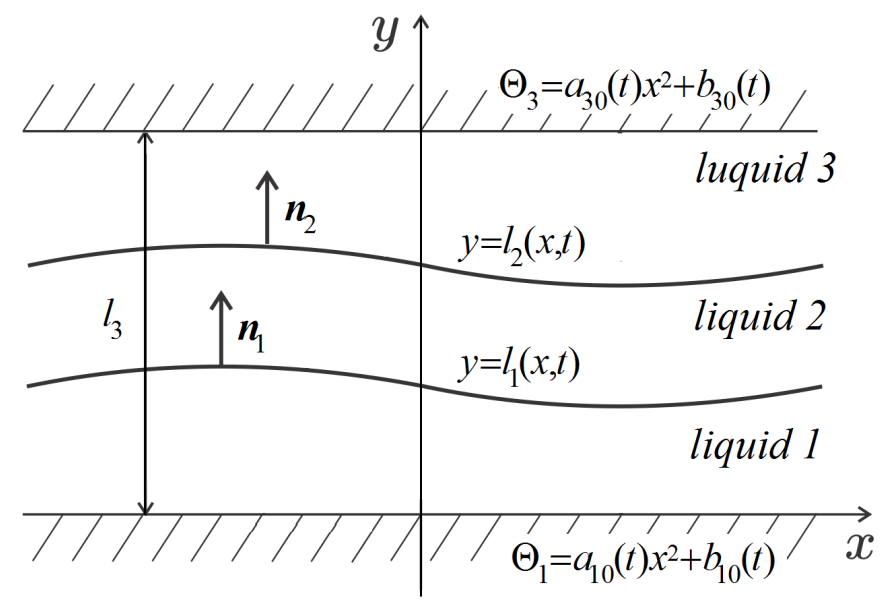

Fig. 1. The domain of the fluids flow

Eqs. (2), (3) we find that, in their domains, the unknowns satisfy the equations

$$
\begin{gathered}
w_{j t}+v_{j} w_{j y}+w_{j}^{2}=\nu_{j} w_{j y y}+f_{j}(t), \\
v_{j t}+v_{j} v_{j y}+d_{j y}=\nu_{j} v_{j y y}, \\
w_{j}+v_{j y}=0 \\
a_{j t}+2 w_{j} a_{j}+v_{j} a_{j y}=\chi_{j} a_{j y y}, \\
b_{j t}+v_{j} b_{j y}=\chi_{j} b_{j y y}+2 \chi_{j} a_{j} .
\end{gathered}
$$

On the interfaces $y=l_{n}(x, t), n=1,2$, there are the conditions

$$
\begin{aligned}
w_{1}\left(l_{1}(x, t), t\right)=w_{2}\left(l_{1}(x, t), t\right), \quad w_{2}\left(l_{2}(x, t), t\right)=w_{3}\left(l_{2}(x, t), t\right), \\
v_{1}\left(l_{1}(x, t), t\right)=v_{2}\left(l_{1}(x, t), t\right), \quad v_{2}\left(l_{2}(x, t), t\right)=v_{3}\left(l_{2}(x, t), t\right), \\
l_{1 t}+x w_{1}\left(l_{1}(x, t), t\right) l_{1 x}=v_{1}\left(l_{1}(x, t), t\right), \quad l_{2 t}+x w_{2}\left(l_{2}(x, t), t\right) l_{2 x}=v_{2}\left(l_{2}(x, t), t\right), \\
a_{1}\left(l_{1}(x, t), t\right)=a_{2}\left(l_{1}(x, t), t\right), \quad a_{2}\left(l_{2}(x, t), t\right)=a_{3}\left(l_{2}(x, t), t\right), \\
k_{1} \frac{\partial a_{1}}{\partial n_{1}}=k_{2} \frac{\partial a_{2}}{\partial n_{1}}, \quad k_{2} \frac{\partial a_{2}}{\partial n_{2}}=k_{3} \frac{\partial a_{3}}{\partial n_{2}}, \\
-406-
\end{aligned}
$$


where $k_{j}>0$ are the thermal conductivities, $\boldsymbol{n}_{n}=\left(-l_{n x}, 1\right) /\left(1+l_{n x}^{2}\right)^{1 / 2}$ are normals to the curves $y=l_{n}(x, t), n=1,2$. Dynamic conditions at the $y=l_{n}(x, t)$ are

$$
\begin{aligned}
& \left(p_{1}-p_{2}\right) \mathbf{n}_{1}+\left[2 \rho_{2} \nu_{2} D\left(\mathbf{u}_{2}\right)-2 \rho_{1} \nu_{1} D\left(\mathbf{u}_{1}\right)\right] \mathbf{n}_{1}=2 \sigma_{1}\left(\Theta_{1}\right) K_{1} \mathbf{n}_{1}+\nabla_{11} \sigma_{1}, \\
& \left(p_{2}-p_{3}\right) \mathbf{n}_{2}+\left[2 \rho_{3} \nu_{3} D\left(\mathbf{u}_{3}\right)-2 \rho_{2} \nu_{2} D\left(\mathbf{u}_{2}\right)\right] \mathbf{n}_{2}=2 \sigma_{2}\left(\Theta_{2}\right) K_{2} \mathbf{n}_{2}+\nabla_{11} \sigma_{2} .
\end{aligned}
$$

In (8), $D$ is the tensor of velocities deformation $\mathbf{u}_{j}=\left(x w_{j}(y, t), v_{j}(y, t)\right)$ and, in the right-hand sides, $\nabla_{11}=\nabla-(\mathbf{n} \cdot \nabla) \mathbf{n}$ denotes the surface gradient, values $K_{n}=l_{n x x}\left(1+l_{n x}^{2}\right)^{-3 / 2}$ are the average curvatures of the interfaces $y=l_{n}(x, t) ; \sigma_{1}\left(\Theta_{1}\right), \sigma_{2}\left(\Theta_{2}\right)$ are surface tension coefficients which depend on the temperature. For the majority of liquid media, the dependence $\sigma_{n}\left(\Theta_{n}\right)$ is well approximated by a linear one

$$
\sigma_{n}\left(\Theta_{n}\right)=\sigma_{n}^{0}-æ_{n} \Theta_{n},
$$

where $æ_{n}>0$ are the temperature coefficients of surfaces tension of lines $y=l_{n}(x, t)$. They are considered constants and determined experimentally.

Dynamic conditions (8) are given in the vector form. Projecting them to the tangential directions $\boldsymbol{\tau}_{n}=\left(1, l_{n x}\right) /\left(1+l_{n x}^{2}\right)^{1 / 2}$, using the dependence (9) and equalities (2) we obtain

$$
\begin{aligned}
& {\left[\mu_{2} D\left(\boldsymbol{u}_{2}\right)-\mu_{1} D\left(\boldsymbol{u}_{1}\right)\right] \boldsymbol{n}_{1} \cdot \boldsymbol{\tau}_{1}=-æ_{1} \nabla_{11} \Theta_{1} \cdot \boldsymbol{\tau}_{1},} \\
& {\left[\mu_{3} D\left(\boldsymbol{u}_{3}\right)-\mu_{2} D\left(\boldsymbol{u}_{2}\right)\right] \boldsymbol{n}_{2} \cdot \boldsymbol{\tau}_{2}=-æ_{2} \nabla_{11} \Theta_{2} \cdot \boldsymbol{\tau}_{2},}
\end{aligned}
$$

where $\mu_{j}=\rho_{j} \nu_{j}$ are the dynamic viscosities of liquids. Projections (8) on the normals $\mathbf{n}_{1,2}$ provide the relations

$$
\begin{gathered}
\rho_{1} d_{1}\left(l_{1}(x, t), t\right)+\frac{\left[\rho_{2} f_{2}(t)-\rho_{1} f_{1}(t)\right] x^{2}}{2}-\rho_{2} d_{2}\left(l_{1}(x, t), t\right)+ \\
\quad+\left[2 \mu_{2} D\left(\boldsymbol{u}_{2}\right)-2 \mu_{1} D\left(\boldsymbol{u}_{1}\right)\right] \boldsymbol{n}_{1} \cdot \boldsymbol{n}_{1}= \\
=\left(\sigma_{1}^{0}-æ_{1}\left[a_{1}\left(l_{1}(x, t), t\right) x^{2}+b_{1}\left(l_{1}(x, t), t\right)\right]\right) l_{1 x x}\left(1+l_{1 x}^{2}\right)^{-3 / 2}, \\
\rho_{2} d_{2}\left(l_{2}(x, t), t\right)+\frac{\left[\rho_{3} f_{3}(t)-\rho_{2} f_{2}(t)\right] x^{2}}{2}-\rho_{3} d_{3}\left(l_{2}(x, t), t\right)+ \\
\quad+\left[2 \mu_{3} D\left(\boldsymbol{u}_{3}\right)-2 \mu_{2} D\left(\boldsymbol{u}_{2}\right)\right] \boldsymbol{n}_{2} \cdot \boldsymbol{n}_{2}= \\
=\left(\sigma_{2}^{0}-\mathfrak{x}_{2}\left[a_{2}\left(l_{2}(x, t), t\right) x^{2}+b_{2}\left(l_{2}(x, t), t\right)\right]\right) l_{2 x x}\left(1+l_{2 x}^{2}\right)^{-3 / 2} .
\end{gathered}
$$

Boundary conditions on the solid walls $y=0, y=l_{3}$

$$
\begin{gathered}
u_{1}(0, t)=0, \quad u_{3}\left(l_{3}, t\right)=0, \quad v_{1}(0, t)=0, \quad v_{3}\left(l_{3}, t\right)=0, \\
a_{1}(0, t)=a_{10}(t), \quad a_{3}\left(l_{3}, t\right)=a_{30}(t)
\end{gathered}
$$

with specified functions $a_{10}(t), a_{30}(t)$. Initial data for the velocities are zero (we study the properties of the solution of problem, which models the motion only under thermocapillarity forces)

$$
u_{j}(y, 0)=0, v_{j}(y, 0)=0,
$$

moreover

$$
l_{n}(x, 0)=l_{n}^{0}(x), a_{j}(y, 0)=a_{j}^{0}(y) .
$$

Note the following features of the problem. It is nonlinear and inverse, since functions $f_{j}(t)$ are unknowns as well. It is easy to understand this, if we exclude the functions $v_{j}(y, t)$ from equations 
of mass conservation. Then the problem reduces to the conjugate problem for functions $w_{j}(y, t)$, $f_{j}(t), a_{j}(y, t)$ и $l_{n}(x, t)$. The problem for functions $b_{j}(y, t)$ separates at the known functions $v_{j}(y, t)$ and $a_{j}(y, t)$, boundary conditions for the functions $b_{j}(y, t)$ are similar to conditions for the functions $a_{j}(y, t)$. The functions $d_{j}(y, t)$ can be restored by quadrature from the second equations (4) up to time functions. So the functions $w_{j}(y, t), v_{j}(y, t) a_{j}(y, t)$ are the solutions of nonlinear parabolic equations with boundary conditions (5)-(7), (12) and initial data (13), (14). The last two conditions in (5) and the fourth in (12) are helpful for determining of the functions $f_{j}(t)$.

We introduce the characteristic scales of length, time and functions $w_{j}, v_{j}, a_{j}, d_{j}, f_{j}$ to simplify the problem (4)-(7), (10)-(14)

$$
l_{1}^{0}, \frac{l_{1}^{02}}{\nu_{1}}, \frac{æ_{1} a^{0} l_{1}^{0}}{\mu_{1}}, \frac{æ_{2} a^{0} l_{1}^{02}}{\mu_{1}}, a^{0}, \frac{æ_{1} a^{0} l_{1}^{0}}{\rho_{1}}, \frac{æ_{1} a^{0}}{\rho_{1} l_{1}^{0}},
$$

respectively, where $l_{1}^{0}=$ const $>0$ is the average value of the layer thickness of the first liquid at $t=0, a^{0}=\max _{t \geqslant 0}\left|a_{30}(t)-a_{10}(t)\right|>0$ or $a^{0}=\max _{j} \max _{y}\left|a_{j}^{0}(y)\right|>0$ when $a_{10}(t)=a_{30}(t)$.

In the dimensionless variables, Marangoni number $M=æ_{1} a^{0} l_{1}^{03} \mu_{1}^{-1} \nu_{1}^{-1}$ appears at the nonlinear summands in eq. (4). The same will be in kinematic conditions (6) at the linear summands containing velocities. It is supposed that temperature coefficients of surface tension are comparable in magnitude $æ_{1} \sim æ_{2}$ and $M \ll 1$. The latter takes place in thin layers or at very high viscosities. Thus, nonlinear summands can be neglected and equation becomes linear. In particular, kinematic conditions take the form $l_{n t}=0$, so $l_{n}=l_{n}(x), n=1,2$.

Let us turn to dynamic conditions (11). After introducing dimensionless variables, in the right side capillary number $C a_{n}=a^{0} l_{1}^{02} æ_{1} / \sigma_{n}^{0}$ appears instead of $\sigma_{1}^{0}$ и $\sigma_{2}^{0}$. In real conditions, for the majority of liquids $C a_{n} \ll 1$, for example, for system water - air $C a \sim 10^{-6}$. Therefore, at such capillary numbers, conditions (11) take the form $l_{n x x}=0$ that is $l_{n}(x)=\alpha_{n} x+l_{n}^{0}$.

Further we assume that $\alpha_{n}=0$ and the surfaces are planes $y=l_{1}^{0}, y=l_{2}^{0}>l_{1}^{0}$, which are parallel to solid walls $y=0, y=l_{3}$; index " 0 " in $l_{n}^{0}$ is omitted.

We can write out the whole linear problem in the dimensional form

$$
\begin{gathered}
w_{j t}=\nu_{j} w_{j y y}+f_{j}(t), \quad j=1,2,3 \\
w_{j}(y, 0)=0, \\
w_{1}(0, t)=0, \quad w_{3}\left(l_{3}, t\right)=0 \\
w_{1}\left(l_{1}, t\right)=w_{2}\left(l_{1}, t\right), \quad w_{2}\left(l_{2}, t\right)=w_{3}\left(l_{2}, t\right), \\
\mu_{2} w_{2 y}\left(l_{1}, t\right)-\mu_{1} w_{1 y}\left(l_{1}, t\right)=-2 æ_{1} a_{1}\left(l_{1}, t\right), \\
\mu_{3} w_{3 y}\left(l_{2}, t\right)-\mu_{2} w_{2 y}\left(l_{2}, t\right)=-2 æ_{2} a_{2}\left(l_{2}, t\right), \\
\int_{0}^{l_{1}} w_{1}(y, t) d y=0, \quad \int_{l_{1}}^{l_{2}} w_{2}(y, t) d y=0, \quad \int_{l_{2}}^{l_{3}} w_{3}(y, t) d y=0,
\end{gathered}
$$

where $0<y<l_{1}$ for $j=1, l_{1}<y<l_{2}$ for $j=2$ and $l_{2}<y<l_{3}$ for $j=3$. Conditions (19) follow from (10), because $\boldsymbol{\tau}_{1}=\boldsymbol{\tau}_{2}=(1,0)$ and $\nabla_{11} \Theta_{1,2} \cdot \boldsymbol{\tau}_{1,2}=2 a_{1,2} x$, according to the expression for the temperature (3). The first two equalities in (2) follow from kinematic conditions (6) and the last one is the no slip condition $v_{3}\left(l_{3}, t\right)=0$.

We write the problem for the functions $a_{j}(y, t)$ as follows

$$
a_{j t}=\chi_{j} a_{j y y}
$$




$$
\begin{gathered}
a_{j}(y, 0)=a_{j}^{0}(y), \\
a_{1}(0, t)=a_{10}(t), \quad a_{3}\left(l_{3}, t\right)=a_{30}(t), \\
a_{1}\left(l_{1}, t\right)=a_{2}\left(l_{1}, t\right), \quad a_{2}\left(l_{2}, t\right)=a_{3}\left(l_{2}, t\right), \\
k_{1} a_{1 y}\left(l_{1}, t\right)=k_{2} a_{2 y}\left(l_{1}, t\right), \quad k_{2} a_{2 y}\left(l_{2}, t\right)=k_{3} a_{3 y}\left(l_{2}, t\right) .
\end{gathered}
$$

\section{Stationary flow}

Problem (15)-(25) has a steady state, according to which the stationary flow of three immiscible, incompressible viscous fluids are described by formulas

$$
\begin{aligned}
& a_{1}^{s}(\xi)=\tilde{a} \xi+a_{10}^{s}, \quad 0<\xi=y / l_{1}<1, \\
& a_{2}^{s}(\xi)=\tilde{a}\left((\xi-1) \bar{k}_{1}+1\right)+a_{10}^{s}, \quad 1<\xi<1 / \bar{l}_{1}, \\
& a_{3}^{s}(\xi)=\bar{k}_{1} \bar{k}_{2} \tilde{a}\left(\xi-\frac{\bar{l}_{2}}{\bar{l}_{1}}\right)+a_{30}^{s}, \quad 1 / \bar{l}_{1}<\xi<\bar{l}_{2} / \bar{l}_{1}, \\
& \bar{w}_{1}^{s}(\xi)=\frac{\bar{l}_{1}\left(\bar{l}_{1}-1\right)}{m_{1}}\left(2 \xi-3 \xi^{2}\right)\left[M_{1}-\frac{m_{5}}{m_{4}}\left(\bar{l}_{1}-1\right)\right], \quad 0<\xi=y / l_{1}<1, \\
& \bar{w}_{2}^{s}(\xi)=-\left[\frac{3 \bar{l}_{1} m_{5}}{m_{4}}(\xi-1)^{2}+\frac{\bar{l}_{1}\left(\bar{l}_{1}-1\right)\left(1+4 \bar{\mu}_{1}(\xi-1)\right)}{m_{1}}\left(M_{1}-\frac{m_{5}\left(\bar{l}_{1}-1\right)}{m_{4}}\right)+2 M_{1}(\xi-1)\right], \\
& 1<\xi<1 / \bar{l}_{1}, \\
& \bar{w}_{3}^{s}(\xi)=2 \bar{\mu}_{2}\left(\xi-\frac{\bar{l}_{2}}{\bar{l}_{1}}\right)\left[-\frac{3 m_{6} \bar{l}_{1}}{4\left(\bar{l}_{2}-1\right)}\left(\xi+\frac{\bar{l}_{2}-2}{\bar{l}_{1}}\right)+\frac{m_{2} m_{5}\left(\bar{l}_{1}-1\right)}{m_{1} m_{4}}-\frac{M_{1} \bar{l}_{1}^{2}}{m_{1}}-M_{2}\right], \\
& 1 / \bar{l}_{1}<\xi<\bar{l}_{2} / \bar{l}_{1} \text {, } \\
& f_{1}^{s}=\frac{6 \bar{l}\left(\bar{l}_{1}-1\right)}{m_{1}}\left(M_{1}-\frac{m_{5}}{m_{4}}\left(\bar{l}_{1}-1\right)\right), \quad f_{2}^{s}=\frac{6 \bar{l}_{1} m_{5}}{\bar{\nu}_{1} m_{4}}, \quad f_{3}^{s}=\frac{3 \bar{l}_{1} \bar{\nu}_{2} \bar{\mu}_{2} m_{6}}{\bar{\nu}_{1}\left(\bar{l}_{2}-1\right)},
\end{aligned}
$$

where $\bar{w}_{j}^{s}=w_{j}^{s} \nu_{1}^{-1} \bar{l}_{1}^{2}, \tilde{a}=\bar{l}_{1}\left(a_{30}^{s}-a_{10}^{s}\right) / m$, functions $a_{j}^{s}, w_{j}^{s}, f_{3}^{s}$ are the stationary solutions of problems $(21)-(25),(15)-(20), a_{10}^{s}, a_{30}^{s}$ are the constant values on the walls $y=0$ and $y=l_{3}$ respectively, $\bar{l}_{1}=l_{1} / l_{2}, \bar{l}_{2}=l_{3} / l_{2}, \bar{k}_{n}=k_{n} / k_{n+1}, \bar{\mu}_{n}=\mu_{n} / \mu_{n+1}, \bar{\nu}_{1}=\nu_{1} / \nu_{2}, \bar{\nu}_{2}=\nu_{3} / \nu_{2}$, $M_{n}=æ_{n} a_{n}^{s}\left(l_{n}\right) \nu_{1}^{-1} \mu_{2}^{-1}$ are the Marangoni numbers and constants $m, m_{1}, m_{2}, m_{3}, m_{4}, m_{5}, m_{6}$ are calculated by the formulas

$$
\begin{gathered}
m=\bar{l}_{1}+\left(1-\bar{l}_{1}\right) \bar{k}_{1}+\left(\bar{l}_{2}-1\right) \bar{k}_{1} \bar{k}_{2}, \quad m_{1}=\bar{l}_{1}^{2}-2 \bar{\mu}_{1} \bar{l}_{1}\left(\bar{l}_{1}-1\right), \\
m_{2}=3 \bar{l}_{1}^{2}-4 \bar{\mu}_{1} \bar{l}_{1}\left(\bar{l}_{1}-1\right), \quad m_{3}=\bar{l}_{1}^{2}-\bar{\mu}_{1} \bar{l}_{1}\left(\bar{l}_{1}-1\right) \\
m_{4}=m_{2} \bar{\mu}_{2}\left(\bar{l}_{2}-1\right)-4 m_{3}\left(\bar{l}_{1}-1\right) \\
m_{5}=\frac{\bar{\mu}_{2}\left(\bar{l}_{2}-1\right)\left(M_{1} \bar{l}_{1}^{2}+M_{2} m_{1}\right)}{\bar{l}_{1}-1}-2 M_{1} \bar{l}_{1}^{2} \\
m_{6}=\frac{m_{2} m_{5}}{m_{1} m_{4}}\left(\bar{l}_{1}-1\right)-\frac{M_{1} \bar{l}_{1}^{2}}{m_{1}}-M_{2} .
\end{gathered}
$$




\section{Non-stationary motion}

To describe the non-stationary motion of three viscous thermally conducting liquids, the Laplace transformation is applied to problems (15)-(20), (21)-(25) (assuming that initial data (22) are zero). As a result, we come to boundary value problem for images $\hat{a}_{j}(y, p)$ of functions $a_{j}(y, t)$

$$
\begin{gathered}
p \chi_{j}^{-1} \hat{a}_{j}(y, p)-\hat{a}_{j y y}(y, p)=0, \\
\hat{a}_{1}(0, p)=\hat{a}_{10}(p), \quad \hat{a}_{3}\left(l_{3}, p\right)=\hat{a}_{30}(p), \\
\hat{a}_{1}\left(l_{1}, p\right)=\hat{a}_{2}\left(l_{1}, p\right), \quad \hat{a}_{2}\left(l_{2}, p\right)=\hat{a}_{3}\left(l_{2}, p\right), \\
k_{1} \hat{a}_{1 y}\left(l_{1}, p\right)=k_{2} \hat{a}_{2 y}\left(l_{1}, p\right), \quad k_{2} \hat{a}_{2 y}\left(l_{2}, p\right)=k_{3} \hat{a}_{3 y}\left(l_{2}, p\right)
\end{gathered}
$$

and images $\hat{w}(y, p)$ of functions $w(y, t)$

$$
\begin{gathered}
p \nu_{j}^{-1} \hat{w}_{j}(y, p)-\hat{w}_{j y y}(y, p)=\nu_{j}^{-1} \hat{f}_{j}(p), \\
\hat{w}_{1}(0, p)=0, \quad \hat{w}_{3}\left(l_{3}, p\right)=0 \\
\hat{w}_{1}\left(l_{1}, p\right)=\hat{w}_{2}\left(l_{1}, p\right), \quad \hat{w}_{2}\left(l_{2}, p\right)=\hat{w}_{3}\left(l_{2}, p\right), \\
\mu_{2} \hat{w}_{2 y}\left(l_{1}, p\right)-\mu_{1} \hat{w}_{1 y}\left(l_{1}, p\right)=-2 \Re_{1} \hat{a}_{1}\left(l_{1}, p\right), \\
\mu_{3} \hat{w}_{3 y}\left(l_{2}, p\right)-\mu_{2} \hat{w}_{2 y}\left(l_{2}, p\right)=-2 \Re_{2} \hat{a}_{2}\left(l_{2}, p\right), \\
\int_{0}^{l_{1}} \hat{w}_{1}(y, p) d y=0, \quad \int_{l_{1}}^{l_{2}} \hat{w}_{2}(y, p) d y=0, \int_{l_{2}}^{l_{3}} \hat{w}_{3}(y, p) d y=0 .
\end{gathered}
$$

In condition (28) and equation $(31), \hat{a}_{10}(p), \hat{a}_{30}(p), \hat{f}_{j}(p)$ are images of functions $a_{10}(t), a_{30}(t), f(t)$ respectively.

The solutions of problems (27)-(30), (31)-(35) can be written as

$$
\begin{gathered}
\hat{a}_{j}(y, p)=d_{j}^{1} \operatorname{sh} \sqrt{\frac{p}{\chi_{j}}} y+d_{j}^{2} \operatorname{ch} \sqrt{\frac{p}{\chi_{j}}} y \\
\hat{w}_{j}(y, p)=c_{j}^{1} \operatorname{sh} \sqrt{\frac{p}{\nu_{j}}} y+c_{j}^{2} \operatorname{ch} \sqrt{\frac{p}{\nu_{j}}} y+\frac{\hat{f}_{j}(p)}{p} .
\end{gathered}
$$

The values $d_{j}^{1}, d_{j}^{2}, c_{j}^{1}, c_{j}^{2}$ and $\hat{f}_{j}(p)$ are determined from the boundary conditions (28)-(30), (32)(35). The type of these values is not presented here because of its complexity.

Remark 1. The solution for the functions $a_{j}(y, t)$ was obtained for the zero initial condition (22). Since the problem (21)-(25) is linear, this problem can be solved for non-zero conditions and when conditions (23) are uniform, boundary conditions (24), (25) remain unchanged and equation (21) has the form $p \hat{a}_{j}(y, p)-\chi_{j}^{-1} \hat{a}_{j y y}(y, p)-a_{j}^{0}(y)=0$.

Let us assume that $\lim _{t \rightarrow \infty} a_{k 0}(t)=a_{k 0}^{s}, k=1,3$. Using formulas (36) and expressions for the values $d_{j}^{1}, d_{j}^{2}, c_{j}^{1}, c_{j}^{2}, \hat{f}_{j}(p)$ we can prove the limit equalities

$$
\begin{gathered}
\lim _{p \rightarrow 0} p \hat{a}_{j}(y, p)=a_{j}^{s}(y), \lim _{p \rightarrow 0} p \hat{w}_{j}(y, p)=w_{j}^{s}(y) \\
\lim _{p \rightarrow 0} p \hat{f}_{j}(p)=f_{j}^{s}
\end{gathered}
$$

where $a_{j}^{s}(y), w_{j}^{s}(y), f_{j}^{s}$ is determined by formulas (26). 


\section{Numerical results}

Let us apply the numerical method of the inverse Laplace transformation to obtained formulas (36). It is enough to show only the pictures for velocities, because they have zeal physical inrepretation. All numerical calculations were made for the system of liquid silicon - water - air (thickness of the layers is the same and equal to $1 \mathrm{~mm}$ ). The corresponding values of the defining parameters are given in Tab. 1.

Table 1. Physical properties of liquids

\begin{tabular}{llll}
\hline Item & liquid silicon & water & air \\
\hline$\rho, \mathrm{kg} / \mathrm{m}^{3}$ & 956 & 998 & 1.205 \\
$\nu \times 10^{-6}, \mathrm{~m}^{2} / s$ & 10.2 & 1.004 & 15.11 \\
$k, \mathrm{~kg} \cdot \mathrm{m} / \mathrm{s}^{3} \cdot K$ & 0.133 & 0.597 & 0.00257 \\
$\chi \times 10^{-6}, \mathrm{~m}^{2} / \mathrm{s}$ & 0.0675 & 0.143 & 21 \\
$æ \times 10^{-5}, \mathrm{~kg} / \mathrm{s}^{2} \cdot K$ & 6.4 & 15.14 & - \\
\hline
\end{tabular}

Figs. 2, 3 show the profiles of the dimensionless functions $\bar{w}_{j}^{s}(\xi)$ (see 26) and transverse velocity $\bar{v}_{j}^{s}(\xi)$ for the case when $M_{1}=M_{2}=0.0005$. Expressions for the velocities $\bar{v}_{j}^{s}(\xi)=v_{j}^{s} \nu_{1}^{-1} l_{1}$ were found from the equation of mass conservation (the third equation in (4))

$$
\begin{gathered}
v_{1}(y, t)=-\int_{0}^{y} w_{1}(y, t) d y, \quad v_{2}(y, t)=-\int_{l_{1}}^{y} w_{2}(y, t) d y \\
v_{3}(y, t)=-\int_{l_{2}}^{y} w_{3}(y, t) d y .
\end{gathered}
$$

In particular, Fig. 2 shows that the function $\bar{w}_{j}^{s}(\xi)$ is negative close to the interfaces $\xi=1$ and $\xi=\bar{l}_{1}^{-1}=2$, so the reverse flow arises here.

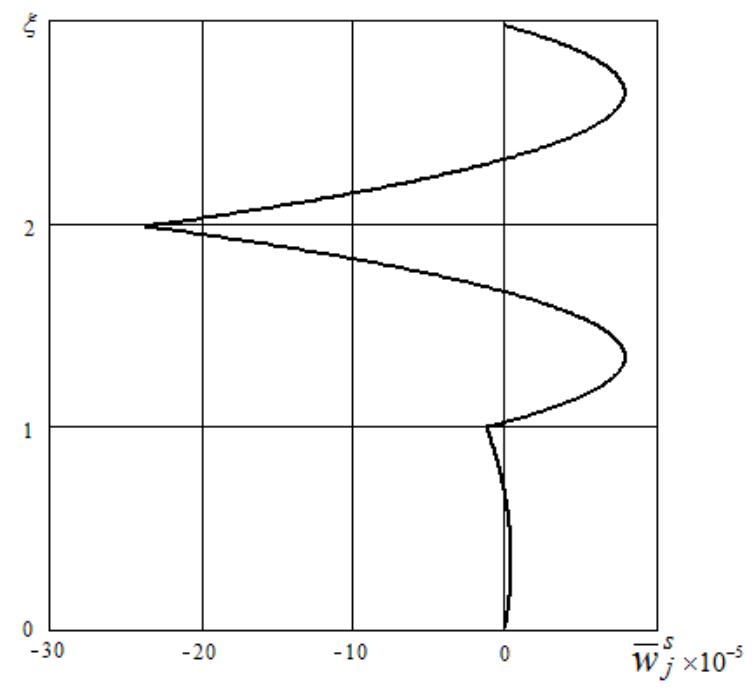

Fig. 2. The stationary profile of function $\bar{w}_{j}^{s}(\xi)$ 


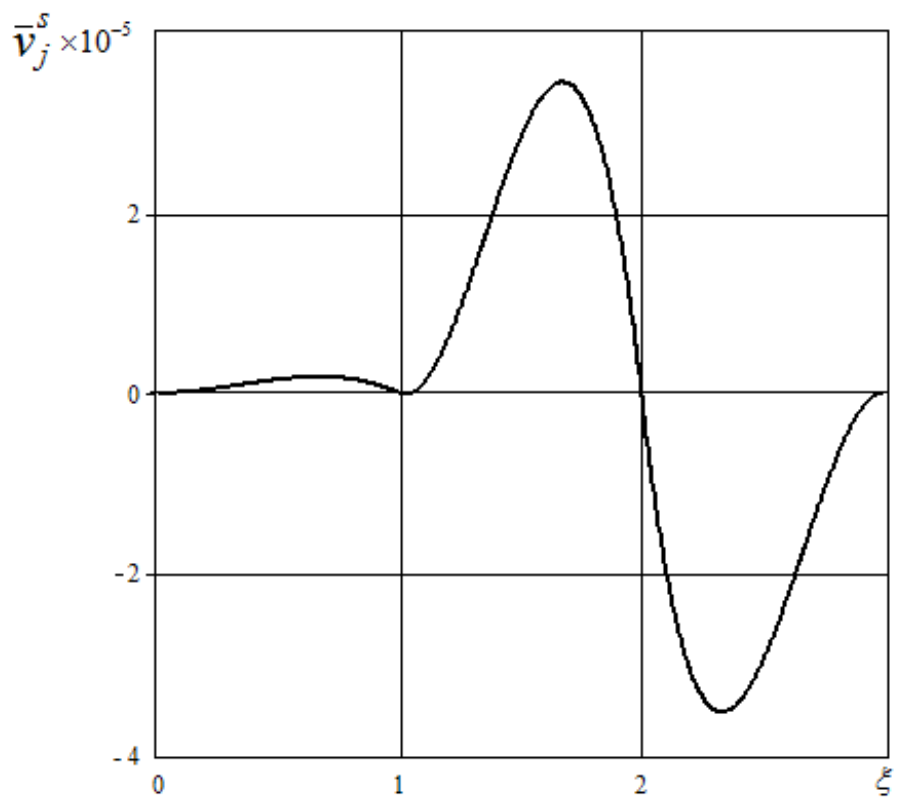

Fig. 3. Stationary profile of transverse velocity $\bar{v}_{j}^{s}(\xi)$

Fig. 4 shows the velocity field in the layers and Fig. 5 shows the velocity field close of the interfaces $\xi=1$ and $\xi=2$. Since $\bar{a}_{1}^{s}(1), \bar{a}_{2}\left(\bar{l}_{1}^{-1}\right)>0\left(\bar{a}_{1}^{s}(1)=79.7, \bar{a}_{2}\left(\bar{l}_{1}^{-1}\right)=33.1\right)$, the temperature field at $x=0$ has a minimum (see (3)). Consequently, in the axial $x$ direction temperature increases and the surface tension decreases (see (9)), so reverse flow occurs close to the interfaces.

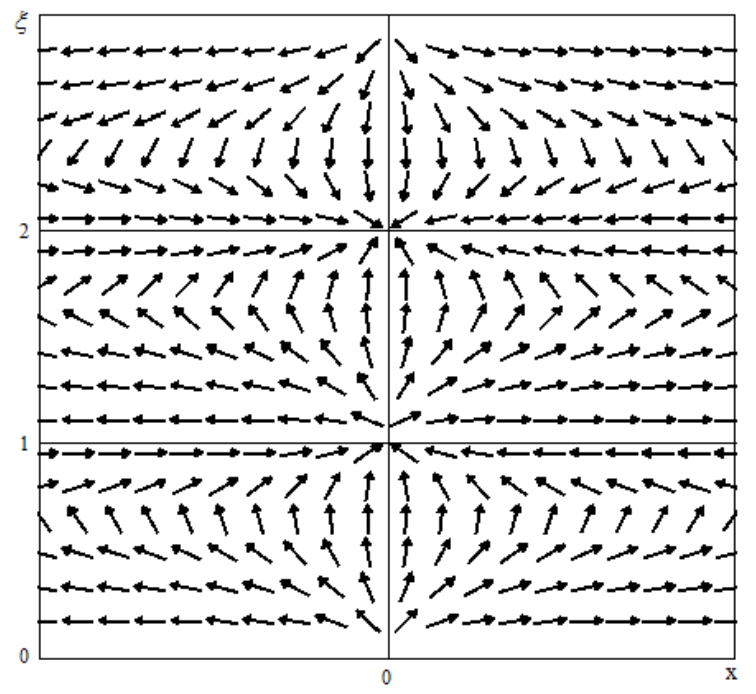

Fig. 4. The velocities field

Fig. 6 shows the convergence evolution of functions $\bar{w}_{j}(\xi, \tau)$ and transverse velocity $\bar{v}_{j}(\xi, \tau)$ to the stationary regime for the case when $\bar{a}_{1}(0, \tau)=1+5 e^{-0.05 \tau} \sin (\tau), \bar{a}_{3}(3, \tau)=0, \tau=\nu_{1} l_{1}^{-1} t$ 
is the dimensionless time (in our case, the real time $t=10^{2} \tau$ ). As it is seen in the pictures, at $\tau=800$ curves almost coincide with the stationary solution. Fig. 7 shows the results of calculations when $\bar{a}_{1}(0, \tau)=2 \sin (0.01 \tau), \bar{a}_{3}(3, \tau)=0$. That is the limit of $\bar{a}_{1}(0, \tau)$ at $\tau \rightarrow \infty$ does not exist and, as it can be seen from the figure, the velocity field does not converge to the stationary one.
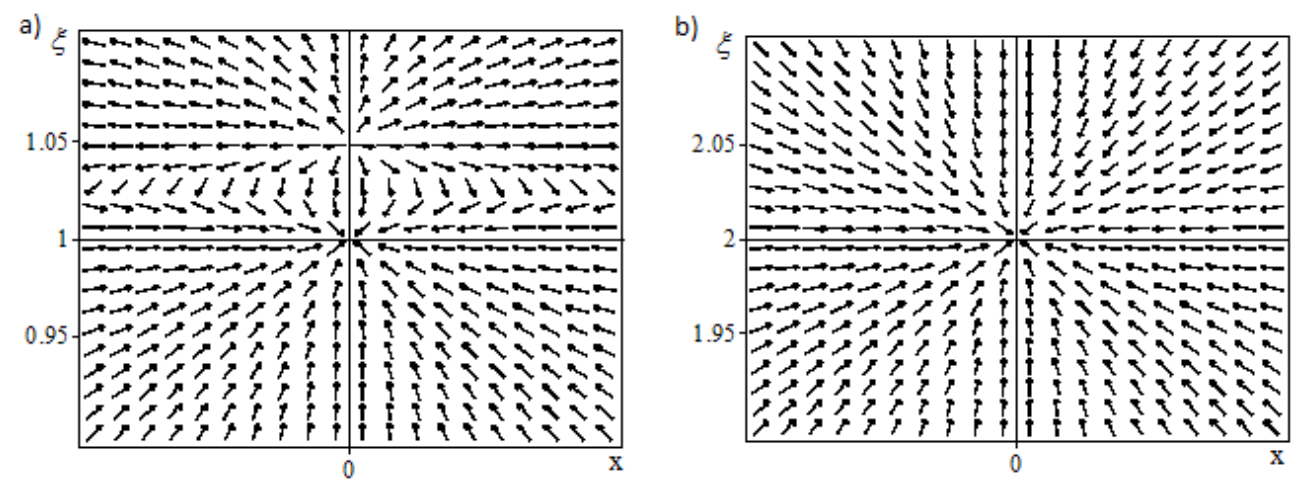

Fig. 5. The velocities field close to the interfaces $\xi=1(a)$ и $\xi=\bar{l}_{1}^{-1}=2(b)$
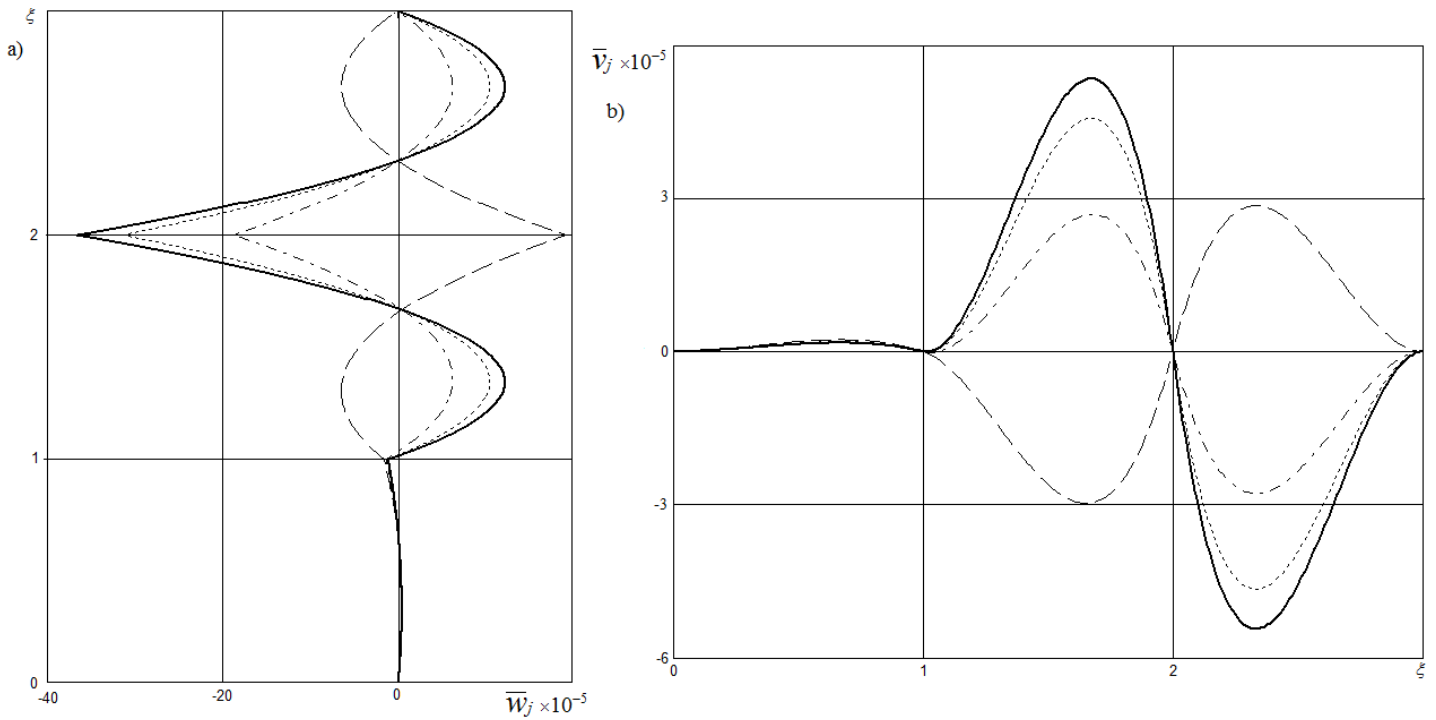

Fig. 6. The convergence evolution of functions $\bar{w}_{j}(\xi, \tau)(\mathrm{a})$ and transverse velocity $\bar{v}_{j}(\xi, \tau)(\mathrm{b})$ to the stationary regime: $\bar{a}_{1}(1, \tau)=1+5 e^{-0.05 \tau} \sin (\tau), \bar{a}_{3}(3, \tau)=0$, stationary regime $(-)$, $\tau=24(--), \tau=400(-\cdot-), \tau=800(\cdots)$

This research was supported by the Russian Foundation for Basic Research (14-01-00067). 

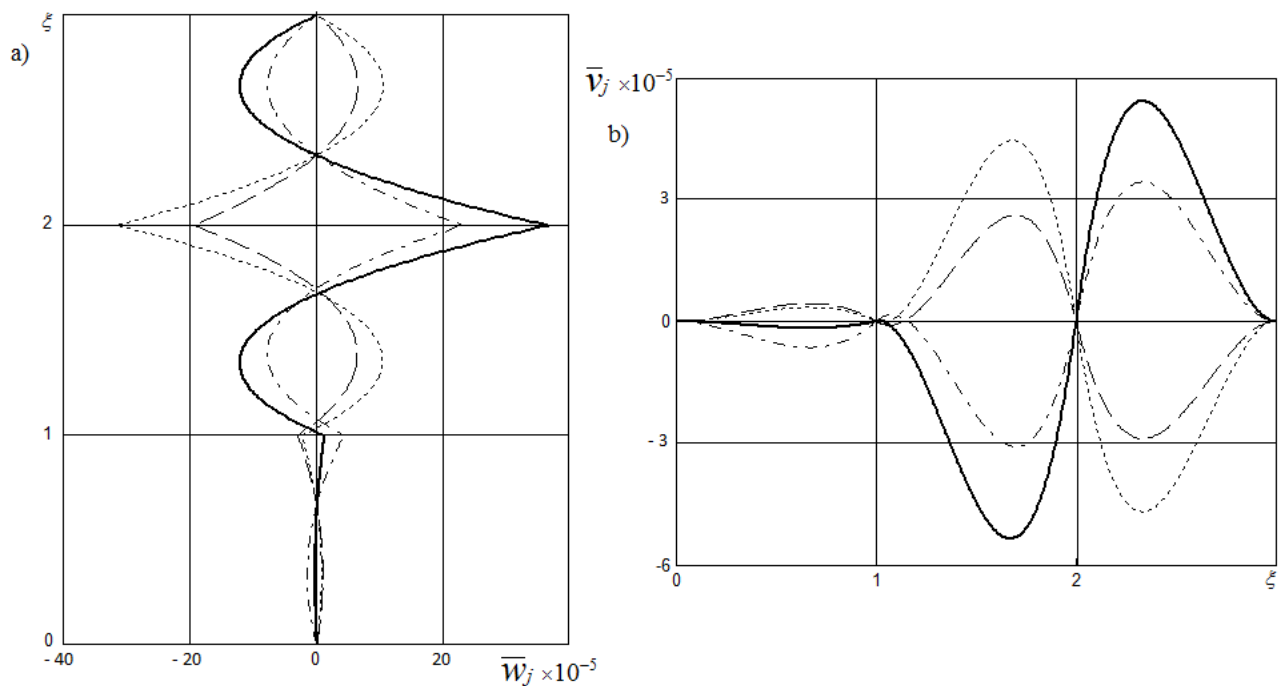

Fig. 7. The dimensionless profiles of functions $\bar{w}_{j}(\xi, \tau)(\mathrm{a})$ and transverse velocity $\bar{v}_{j}(\xi, \tau)(\mathrm{b})$ : $\bar{a}_{1}(1, \tau)=2 \sin (0,01 \tau), \bar{a}_{3}(3, \tau)=0$, stationary regime $(-), \tau=470(--), \tau=850(-\cdot-)$, $\tau=1070(\cdots)$

\section{References}

[1] V.K.Andreev, V.E.Zahvataev, E.A.Ryabitskii, Thermocapillary Instability, Nauka, Novosibirsk, 2000 (in Russian).

[2] A.Nepomnyashii, I.Simanovskii, J.-C.Legros, Interfacial Convection in Multilayer System, Springer, New-York, 2006.

[3] R.Narayanan, D.Schwabe, Interfacial Fluid Gynamics and Transport Processes, SpringerVerlag, Berlin, Heidelberg, New-York, 2003.

[4] R.Kh.Zeytovnian, Convection in Fluids, Springer, Dordrecht, Heidelberg, London, NewYork, 2009.

[5] V.K.Andreev, The Birikh Solution of Convection Equations and it Some Generalization, Preprint no. 1-10, Institut vychislitel'nogo modelirovaniya SB RAS, Krasnoyarsk, 2010.

[6] V.K.Andreev, O.V.Kaptsov, V.V.Pukhnachov, A.A. Radionov, Applications of GroupTheoretical Methods in Hydrodynamics, Kluwer Academic Publisher, Dordrecht-BostonLondon, 1998.

[7] V.K.Andreev, Y.A.Gaponenko, O.N.Goncharova, V.V.Pukhnachov, Mathematical Models of Convection, Walter de Gruyter GmbH and Co. KG, Berlin-Boston, 2012.

[8] L.G.Napolitano, Plane Marangoni-Poiseuille flow two immiscible fluids, Acta Astronautica, 7(1980), no. $4-5,461-478$

[9] V.K.Andreev, V.B.Bekezhanova, Stability of Non-isothermal Fluids, J. of Siberian Federal University, Mathematics and Physics, 4(2011), no. 4, 434-444 (in Russian). 
[10] V.K.Andreev, On a conjugate initial boundary value problem, Diff. equations, (2008), no. 5, $1-7$.

[11] V.K.Andreev, V.B.Bekezhanova, Stability of Non-isothermal Fluids (Review), J. Appl. Mech. and Tech. Phys., 54(2013), no. 2, 171-184.

[12] V. K.Andreev, E.N.Lemeshkova, Evolution of the thermocapillary motion of three liquids in a plane layer, Appl. Math. and Mech., 78(2014), no. 4, 485-492.

[13] I.V.Denisova, On the problem of thermocapillary convection for two incompressible fluids separated by a closed interface, Prog. Nonlinear Differ. Equ. Appl., 61(2005), 45-64

[14] I.V.Denisova, Thermocapillary convection problem for two compressible immiscible fluids, Microgravity Sci. Technol., 20(2008), no. 3-4, 287-291.

[15] K.Hiemenz, Die Grenzschicht an einem in den gleichförmigen Flüssigkeitsstrom eingetauchten geraden Kreiszylinder, Dinglers Poliytech. J., 326(1911), 321-440.

[16] J.F.Brady, A.Acrivos, Steady flow in a channel or tube with an accelerating surface velocity, J. Fluid Mech., 112(1981), 127-150.

[17] N.N.Bobkov, Yu.P.Gupalo, The structure of the flow in the liquid layer and the spectrum of the boundary value problem for the nonlinear dependence of the surface tension from temperature, Appl. Math. and Mech., 60(1996), no. 6, 1021-1028.

[18] Yu.P.Gupalo, Yu.S.Ryazantsev, On a thermocapillary motion of a fluid with a free surface at the nonlinear dependence of the surface tension from temperature, Fluid Dynamics, (1988), no. $5,132-137$.

[19] Yu.P.Gupalo, Yu.S.Ryazantsev, A.V.Skvortsov, Influence of thermal capillary forces on the fluid flow with free boundary, Fluid Dynamics, (1989), no. 5, 3-7.

\section{Двумерное термокапиллярное движение трех жидкостей в плоском канале}

Виктор К. Андреев

Елена Н. Черемных

Институт вычислительного моделирования СО РАН

Академгородок, 50/44, Красноярск, 660036

Исследовано двумерное ползущее движение трех несмешивающихся несжимаемых вязких теплопроводных жидкостей в плоском канале, ограниченном твердыми неподвижсными стенками, на которых известно распределение температур. В образах по Лапласу построено точное нестационарное решение в виде квадратур и приведены некоторые численные результаты поведения полей скоростей и температур в слоях.

Ключевые слова: термокапиллярность, поверхность раздела, математическое моделирование, численные эксперименты. 